\title{
Relaxation Effect of a Respiration-Leading Stuffed Toy
}

\author{
Hiroki Uratani, ${ }^{*}$ " Mieko Ohsuga ${ }^{* *}$
}

\begin{abstract}
After experiencing natural disasters, accidents, and shocking incidents, some children experience post-traumatic stress disorder (PTSD). A respiration control method that relaxes the body and mind may efficiently improve PTSD symptoms. Therefore, we developed a stuffed toy using two airbags to measure the respiration wave and guide a child's respiration using the up-and-down movements of the toy's abdomen to help them relax. The respiration wave was measured by the sensing device, and the child's respiration could be led by the moving device. We then performed an evaluation experiment. Participants in the experiment consisted of nine healthy girls aged 8-10 years. The results showed that the respiration guidance resulted in fewer variations in the children's mean heart rate than that with just hugging $(p<0.05)$. This result suggests that the respiration-guiding trial relaxes children compared with just hugging the stuffed toy without motion. The effectiveness of this stuffed toy in improving PTSD symptoms in children should be further evaluated.
\end{abstract}

Keywords: relaxation, respiration-leading, children, airbag, stuffed toy.

Adv Biomed Eng. 7: pp. 100-106, 2018.

\section{Introduction}

After experiencing natural disasters, accidents, and shocking incidents, especially after major disasters such as earthquakes, some children as well as adults experience post-traumatic stress disorder (PTSD). These children require mental health care. After the Great Hanshin-Awaji Earthquake in 1995, research was conducted on mental health care for children who experienced fearful events or losses. It was reported that PTSD coping techniques should be prioritized in lower-grade elementary school girls [1]. The respiration method relaxes the mind and body, and is an important PTSD prevention technique [2]. This method is effective and easy to learn. Breathing slowly and deeply is a popular method for coping with stressful events that occur in daily life [3]. Teaching mental care skills as a preventive technique, rather than as a coping mechanism, for disaster-induced mental illnesses is needed [1].

Therefore, we developed a prototype respiration-leading stuffed bear whose abdomen moves up and

Received on April 24, 2017; revised on August 25, 2017, December 18, 2017, and February 13, 2018; accepted on February 14, 2018.

*Neo-Education Science Research Institute, EQWEL Co., Ltd., Osaka, Japan.

** Department of Robotics, Osaka Institute of Technology, Osaka, Japan.

\# 3-15 Kitakawahori-cho, Tennoji-ku, Osaka 543-0053, Japan.

E-mail: uratani@eqwel.net down [4]. The prototype respiration-leading stuffed toy functions as follows. The session starts when the child hugs the stuffed toy and turns the switch on. First, the toy starts to measure the child's respiration period (RP) from the child's pulse data for $30 \mathrm{~s}$. Next, the toy calculates the mean RP and subsequently moves its abdomen up and down at the same frequency as the child's resting RP to guide his/her respiration, because we concluded in our preliminary experiment that it is necessary to begin leading the child's respiration from his/her resting RP [5]. A respiration-leading period (LP) ratio of inhalation and exhalation of $1: 2$ is considered the most relaxing respiration ratio $[6,7]$. The movement of the abdomen slows gradually (i.e. the LP lengthens) to decelerate the child's breathing. The LP lengthens gradually until it reaches $8 \mathrm{~s}$. We examined how the child's respiration was led and how the child relaxed by hugging it. This device was tested on 48 healthy children ( 26 boys and 22 girls) aged 4-12 years. The results showed that children under 7 years and boys were poorly led compared with girls. Their respiration patterns suggested that the final LP (8 s) was too long [4].

Accordingly, we intended to determine the most relaxing RP for children. Data from studies on heart rate variability (HRV) biofeedback training suggest that breathing at an RP that leads to the highest HRV is most effective for improving chronic diseases. There are two components of HRV: the Mayer wave (approximately $10 \mathrm{~s}$ ) and the respiration component (approximately $4 \mathrm{~s}$ ). The HRV amplitude becomes the highest when the RP lengthens, and the respiration component resonates with 
the Mayer wave component. This phenomenon is called "resonance," and the respiratory frequency at which resonance occurs is called the resonance frequency [8]. When a patient breathes at the resonance frequency, mental and physical symptoms of chronic diseases including PTSD [2], depression [9, 10], chronic heart failure [11], hypertension [12], and prehypertension [13] improve. The resonance frequency is reported to be $4.5-$ 7 breaths per minute (i.e. approximately $9-13 \mathrm{~s}$ in a period) in adults [14], but the resonance frequency in children has not been reported. Therefore, we measured the relationship between the RP and physiological indices including HRV in children. The participants were 10 children aged 5-12 years. The highest HRV was at a RP of 10-12 s in all 10 children. However, the most suitable RP for smooth breathing and relaxation was different from that at which HRV is the highest [15]. As a result, we concluded that it is suitable to lengthen the LP until it reaches the most relaxing RP (i.e. not the RP at which HRV is the highest) for the child.

According to these results, we developed a respiration-leading stuffed toy whose abdomen moves up and down.

\section{Development}

The respiration-leading stuffed toy consists of two airbags (size: $115 \times 85 \times 10-50 \mathrm{~mm}$ ), two air pressure sensors (CQ30A-G102, MKT Taisei), two solenoid valves, a pneumatic motor (AJK-B2701, Xiamen AJK Technology), and a micro controller (Arduino Uno, Smart Projects). The components are put into a stuffed toy (Rilakkuma Kuttari stuffed toy, size: $560 \times 360 \times 140 \mathrm{~mm}$, San-X). One airbag is set in the upper abdomen as a respiration-leading airbag (moving device), and the other airbag is set in the lower abdomen as a respiration sensor (sensing device) [16] (Fig. 1).

The algorithm of respiration-leading is as follows. First, the participant's respiration wave in the resting state is measured by the sensing device for $30 \mathrm{~s}$, and the mean RP is calculated. Next, the rhythm of the up-anddown movement of the moving device (LP) starts from that RP and continues at the same rhythm for five RPs. Subsequently, the LP lengthens $0.5 \mathrm{~s}$ per three RPs until the LP reaches $5 \mathrm{~s}$. After that, when the RP synchronizes with the LP for three respirations, the LP lengthens by $0.5 \mathrm{~s}$. In contrast, when the RP does not synchronize with the LP for three respirations, the LP shortens by $0.5 \mathrm{~s}$. The criterion of synchronization is set at a RP within $\pm 0.8 \mathrm{~s}$ of LP. The longest LP is set to $10 \mathrm{~s}$.

We examined how the child's respiration was measured and led by the stuffed toy. Participants in the experiment consisted of 12 healthy girls aged 7-10 years. The results showed that respiration wave could be measured by the sensing device and the child's respiration could be led by the moving device [16].

\section{Methods}

\subsection{Participants}

Nine healthy girls aged 8-10 years participated in the experiment. Informed consent was obtained from the parents, and informed assent was obtained from the children. Before the experiment, we confirmed that the child's condition was not bad and that there was no problem to begin the experiment. This experiment was approved by the Life Science Ethics Committee of Osaka Institute of Technology.

\subsection{Measurements}

Electrocardiogram (ECG) and respiration waveform were obtained during the experiment. The ECG was recorded using electrodes placed on the chest and abdomen of the participant. Data were amplified by a biological amplifier (Polyam4, NIHONSANTEKU). Respiration waveform was recorded from the child's abdomen by the airbag sensor in the stuffed toy [16].

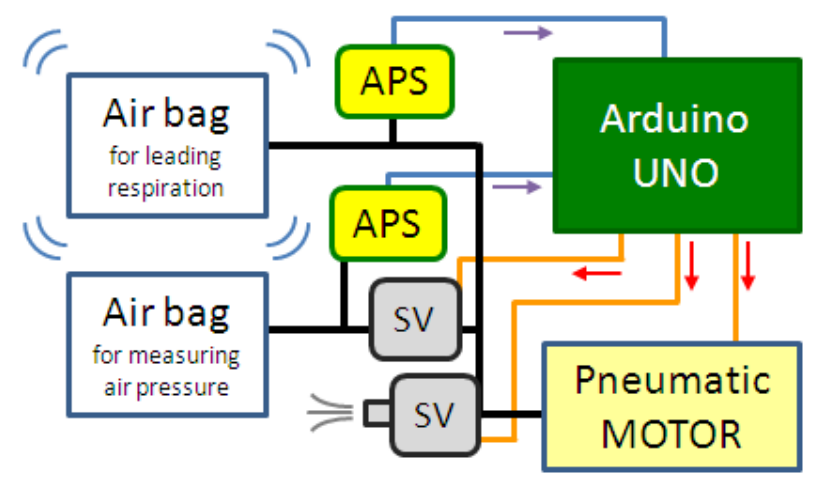

APS: Air pressure sensor; SV: Solenoid valve.

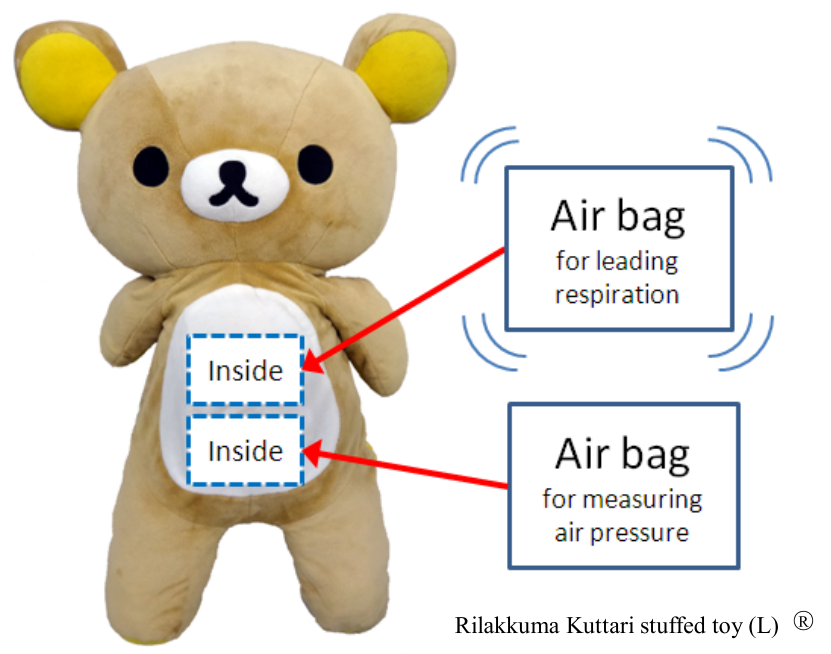

Fig. 1 Components of the device [16] (revised). 


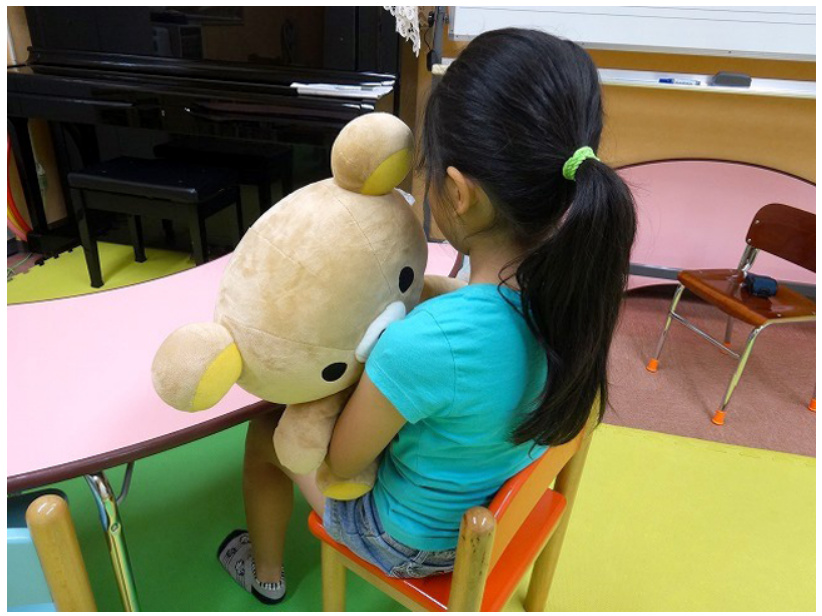

Fig. 2 A scene of an experiment.

\subsection{Experimental protocol}

Before the experiment, all the participants practiced the respiration-leading with the stuffed toy. The respiration-leading practice consisted of measuring the mean RP (30 s) in the resting state and during respiration-leading trial $(180 \mathrm{~s})$ each for three times. The experiment started more than a week after the practice.

At the beginning of the experiment, the participant practiced respiration-leading one more time. After the practice, the ECG was measured for $60 \mathrm{~s}$ while the participant was in a resting state with eyes closed. Subsequently, the participant performed either respiration-leading or hugging (Fig. 2). In this experiment, we did not add a temporary stress task before measuring the ECG. To decrease the burden of the participants, we only measured the difference between the resting state and after the trial.

For the respiration-leading trial, the mean RP of participant's resting state was measured for $30 \mathrm{~s}$. Subsequently, the respiration-leading trial commenced. The participant was instructed to synchronize her respiration with the movement of the abdomen of the stuffed toy for $180 \mathrm{~s}$. The LP of respiration-leading started from the child's resting mean RP and increased automatically up to $5 \mathrm{~s}$. The LP increased by $0.5 \mathrm{~s}$ every three respirations. After reaching $5 \mathrm{~s}$, the LP increased by $0.5 \mathrm{~s}$ when the child's RP synchronized with the LP of the stuffed toy three times in five respirations. In previous studies, the LP only increased when the RP synchronized with the LP of the leading device for three consecutive times [16, 17]. But the LP did not increase as much because this standard was too rigorous for children [16]. Therefore, we eased the standard as mentioned above. The ratio of the inhalation period to the exhalation period of the LP was constant at 1:2 (Fig. 3).

In the hugging trial, the abdomen of the toy did not

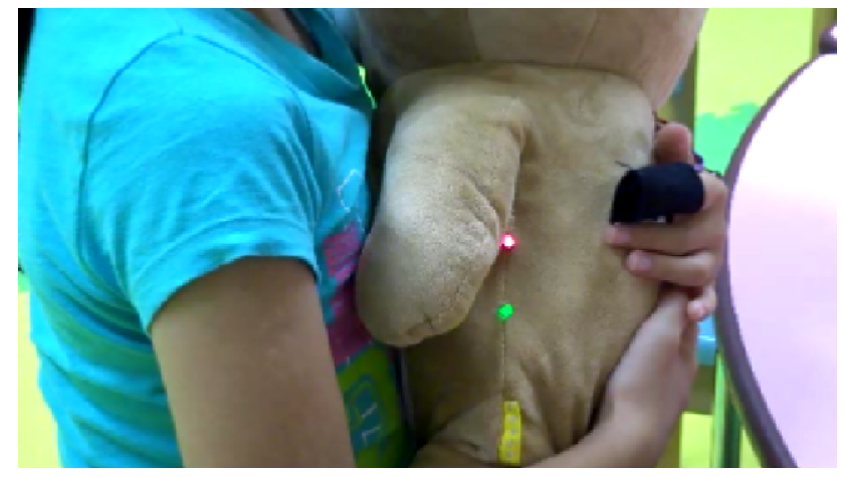

Fig. 3 A scene from a respiration-leading session.

move. The participant was instructed to hug the stuffed toy for $180 \mathrm{~s}$.

At the end of the hugging or the respiration-leading trial, the ECG in the resting state with eyes closed was measured for $60 \mathrm{~s}$. After completing one trial, the participant executed the other one. The order of the trials was randomized. The participant rated the degree of relaxation after both trials, and ease of following the instructions of the stuffed toy after the respiration-leading trial, using a 7-point rating questionnaire. The relaxation scale ranged from low, expressed as "nervous," to high, expressed as "calm." The ease of following instructions ranged from low, expressed as "hard," to high, expressed as "easy."

\subsection{Analysis}

The RP of each respiration during the respiration-leading trial was calculated by a micro-controller in real time. Three parameters were determined from the RPs and LPs of the respiration-leading trial: starting RP, longest LP, and extension of LP. Counting of extension of LP started after the LP reached $5 \mathrm{~s}$. The HR of the resting state during 10-60 s was analyzed from the ECG using MATLAB (The MathWorks). The mean HR variation of each condition was calculated (HR after trial - HR before trial).

The overlap ratio was calculated using the RP and the LP. The RP inhalation period (RPI) and the LP inhalation period (LPI) of each respiration were analyzed. If there was overlap between the RPI and LPI, the overlapped time (A) and the sum time (B) that includes the overlapped portion were determined in seconds. The overlap ratio was calculated as $\mathrm{A} / \mathrm{B} \times 100 \%$ for each respiration and was averaged over a trial. The overlap ratio equals $100 \%$ when the RPI and LPI overlap entirely, and equals $0 \%$ when the RPI and the LPI do not overlap (Figs. 4 and 5) [4]. 


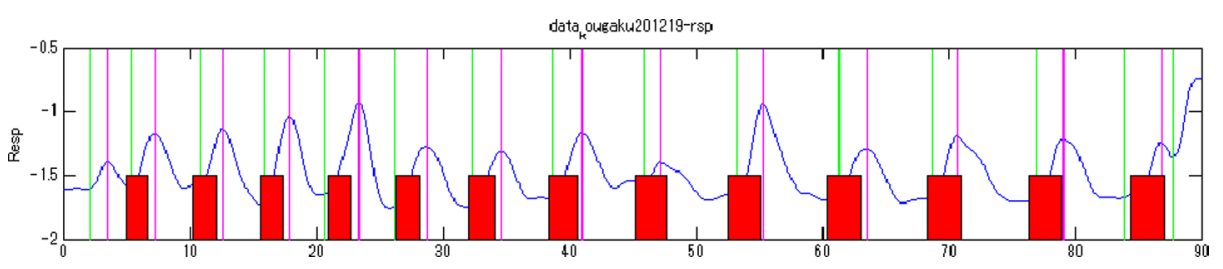

Fig. 4 An example of respiration leading data [4]. Units: seconds; blue lines: respiration data, red rectangles: periods of leading inhalation, green lines: start of inhalation, purple lines: end of inhalation.

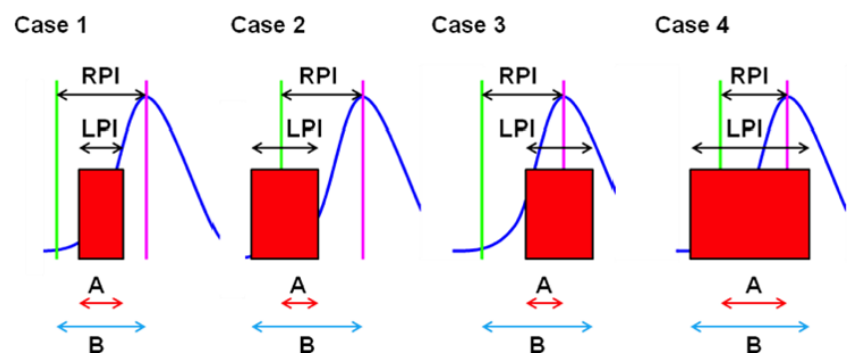

Fig. 5 Illustration of the overlap ratio (A/B) [4].

\section{Results}

\subsection{Respiration-leading period (LP) and overlap ra- tio}

Table 1 summarizes the LP and overlap ratio of nine participants. The mean RP in the resting state (starting RP) was $4.02 \mathrm{~s}$, the mean longest LP was $6.47 \mathrm{~s}$, the mean extension of LP was 2.56 times, and the mean overlap ratio was $53.2 \%(0-180 \mathrm{~s})$.

We examined the participants' respiration data in Table 1 to determine whether their respirations were led or not. We concluded that No. 3 and No. 5 were not led because LP extension was zero and the overlap ratios were lower than 50\%. Moreover, we concluded that No. 9 was not led because the overlap ratio was lower than $50 \%$ even though LP was extended once.

\subsection{Change in mean HR}

Table 2 shows the changes in mean HR in each trial. The order of the two trials was randomized, and the order is shown in the order line. The changes in mean HR of eight of nine participants were smaller (i.e. decline of HR is greater or rise of HR is smaller) in the respiration-leading trial.

We excluded the data of three participants whose respiration was not led from our analysis and compared the changes in mean HR of the six remaining participants. The average change in mean HR in hugging trial was $-0.21 \mathrm{bpm}$. The average change in mean HR in respiration-leading trial was $-3.75 \mathrm{bpm}$. Analysis by paired $\mathrm{t}$-test confirmed that the average change in mean HR was
Table 1 Respiration-leading periods and overlap ratio during 5respiration-leading states.

\begin{tabular}{ccccc}
\hline \hline No. & $\begin{array}{c}\text { Starting } \\
\text { RP (s) }\end{array}$ & $\begin{array}{c}\text { Longest } \\
\text { LP (s) }\end{array}$ & $\begin{array}{c}\text { Extension } \\
\text { (numbers) }\end{array}$ & $\begin{array}{c}\text { Overlap } \\
\text { Ratio } \\
(\%)\end{array}$ \\
\hline 1 & 4.0 & 7.5 & 5 & 52.2 \\
2 & 5.3 & 5.8 & 1 & 75.3 \\
3 & 4.0 & 5.0 & 0 & 20.6 \\
4 & 3.8 & 6.8 & 3 & 59.1 \\
5 & 4.0 & 5.0 & 0 & 44.2 \\
6 & 5.1 & 7.6 & 5 & 55.6 \\
7 & 3.4 & 7.9 & 5 & 77.4 \\
8 & 3.9 & 6.9 & 3 & 60.9 \\
9 & 2.7 & 5.7 & 1 & 33.3 \\
\hline \hline
\end{tabular}

RP: respiration period; LP: respiration-leading period;

Extension: numbers (frequency); Red Nos.: Children who were not led.

Table 2 Changes in mean HR in each trial.

\begin{tabular}{cccccccccc}
\hline \hline No. & 1 & 2 & 3 & 4 & 5 & 6 & 7 & 8 & 9 \\
\hline Hug & -3.7 & 1.4 & 3.9 & 0.4 & $\mathbf{- 3 . 6}$ & 5.7 & -3.3 & -1.8 & 0.4 \\
RL & $\mathbf{- 4 . 2}$ & $\mathbf{- 5 . 9}$ & $\mathbf{- 6 . 8}$ & $\mathbf{- 0 . 4}$ & $\mathbf{- 0 . 3}$ & $\mathbf{1 . 2}$ & $\mathbf{- 4 . 4}$ & $\mathbf{- 8 . 7}$ & $\mathbf{- 2 . 1}$ \\
\hline Order & H-L & L-H & L-H & H-L & H-L & L-H & H-L & L-H & L-H \\
\hline \hline
\end{tabular}

Italic bold number: trial with relatively smaller change; Hug: hugging trial; RL: respiration-leading trial;

Order: order of trials; H-L: Hug $\rightarrow$ RL; L-H: RL $\rightarrow$ Hug

significantly smaller in respiration-leading trial $[p=$ 0.0402] (Fig. 6).

\subsection{Subjective measurements}

Table 3 illustrates the subjective rating of nine participants. Six participants answered that they were more relaxed after the hugging trial. Two participants answered that they were more relaxed after the respiration-leading trial. One participant (No. 8) answered that she was 


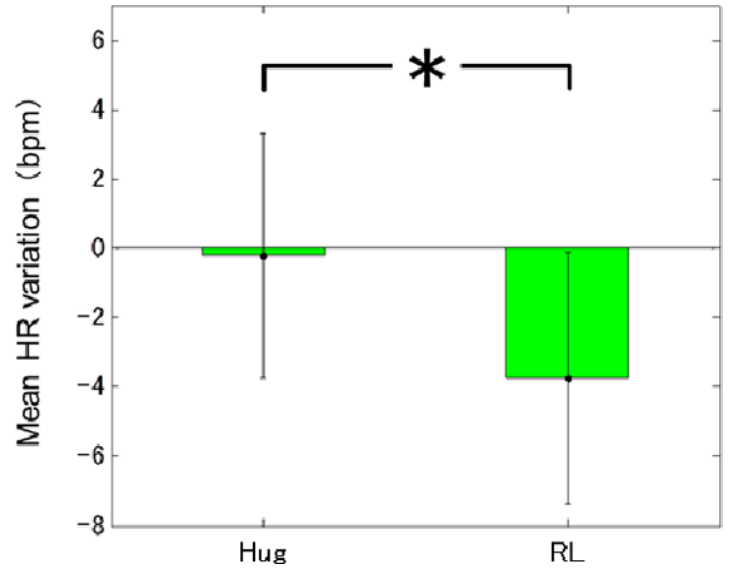

Fig. 6 Comparison of the average change in mean HR between two trials ( 6 children).

$\left.{ }^{*} p<0.05\right)$ HR: heart rate, Hug: hugging trial, $\mathrm{RL}$ : respiration-leading trial.

Table 3 Subjective rating of relaxation and ease in following respiration-leading instruction.

\begin{tabular}{|c|c|c|c|c|c|c|c|c|c|c|}
\hline \multicolumn{2}{|c|}{ No. } & 1 & 2 & 3 & 4 & 5 & 6 & 7 & 8 & 9 \\
\hline \multirow{2}{*}{ Relax } & Hug & 7 & 7 & 3 & 5 & 6 & 7 & 6 & 7 & 6 \\
\hline & RL & 5 & 6 & 7 & 6 & 5 & 6 & 5 & 7 & 5 \\
\hline Ease & $\mathrm{RL}$ & 5 & 7 & 4 & 5 & 4 & 7 & 5 & 6 & 6 \\
\hline
\end{tabular}

Relax: Subjective measure of relaxation;

italic bold number: Comparatively relaxed condition;

Ease: Subjective measure of ease in following respiration-leading;

Hug: Hug condition; RL: Respiration-Leading condition; units: scores on a scale of 1-7.

equally relaxed after both trials. Seven participants rated five points or more regarding the ease in following respiration-leading instructions. Two participants (No. 3 and No. 5) with 0 extension rated 4 points regarding the ease in following respiration-leading instructions.

\section{Discussion}

We developed a portable respiration-leading stuffed toy and measured the relaxation effect using mean heart rate in nine healthy girls.

First, we examined the variation of LP during the respiration-leading session. There were two participants (No. 3 and No. 5) whose LP did not extend in the experiment. This suggests that their respiration was not led. Several reasons may explain this phenomenon: (1) the abdomen of the stuffed toy and the abdomen of the child were not properly in contact, which may have hindered the device from retrieving exact respiration measures; (2) the child could not synchronize her respiration with the respiration-leading, but continued breathing at her own pace. Indeed, there were two participants (No. 2 and No. 9) whose LP was extended only once during the experiment. Examining their overlap ratios, one participant (No. 2) displayed a high ratio exceeding $70 \%$, while the other participant (No. 9) displayed a low ratio below $40 \%$. Following the experiment, we found that the respiration of the former participant was indeed led, but the device failed to extend the LP due to a bug in the algorithm for LP extension. In contrast, when examining the wave-form of the latter participant (No. 9), we noted that her respiration was led only during the first half and the LP was extended once. However, during the second half, she could not synchronize her respiration with the respiration-leading device and breathed at her own pace. Therefore, we considered that the respiration of participant 9 was not led.

We excluded three participants whose respiration was not led, and compared the relaxation effect in the six remaining participants. When comparing between the two trials, we observed that the average change in mean HR was significantly smaller in the respiration-leading trial. The smaller change in mean HR reflects a relaxed participant. These findings suggest that the relaxation effect of the respiration-leading trial was higher than that of the hugging trial in the six participants whose respiration was led.

In two (No. 3 and No. 9) of the three participants whose respirations were not led, the change in mean HR was smaller in the respiration-leading trial compared with the hugging trial. Upon examining the respiration wave-form of participant No. 3, we concluded that the LP extension corresponded to 0 , because RP was not measured correctly by the device. Therefore, there is a possibility that the respiration of No. 3 was led after all. Moreover, the respiration of No. 9 was led in the first half as mentioned above, thus resulting in a smaller change in mean HR in the respiration-leading trial. The respiration of No. 6 was led, and as we hypothesized, the change in mean HR was smaller in the respiration-leading trial. However, the change in mean HR was also a positive value in the respiration-leading trial. This phenomenon can be interpreted as that the participant was physiologically stressed due to her excessive attempt to synchronize her respiration with the device.

In an experiment using a human-shaped cushion called "Hugvie" that functioned as a mobile phone, participants who had a conversation with a stranger while hugging Hugvie were more relaxed than participants who went through the same procedure with only a mobile phone instead of Hugvie [18]. In fact, we could confirm this phenomenon with almost half of our participants; four of nine showed negative value for the change in mean HR in the hugging trial. However, the average 
change in mean HR was smaller in the respiration-leading trial. These results suggest that a combination of hugging and respiration-leading increases the relaxation effect.

Although the physiological indices showed a higher relaxation effect in the respiration-leading trial, six of nine participants responded that they felt more relaxed after the hugging trial. Moreover, four of six participants whose respirations were led also confirmed that they were more relaxed after the hugging trial. These results suggest that children do not rate their physiological changes objectively, as observed in our previous study [5, 15]. Although they were physiologically relaxed, it is plausible that the children felt more stressed than they were in reality while performing under the unfamiliar circumstances of the present experiment. Given that children often misconceive their physiological and psychological states, we suggest that when guided to be relaxed, children need feedback information regarding the degree of physiological relaxation.

Two participants (No. 3 and No. 5) whose extension time was $0 \mathrm{~s}$ rated 4 points when asked about the ease in following respiration-leading instructions. This suggests that they could perceive synchronization of respiration objectively.

Although the respiration-leading stuffed toy had functions including displaying the states of pulse rate and inspiration by the blink of LED, and setting the LP longer when the respiration of participant synchronizes with the respiration-leading of the device, we did not instruct the children to be aware of this feedback information. Therefore, there is a possibility that the relaxation effect and the accuracy of respiration-leading may be higher if participants are instructed about this feedback information.

\section{Conclusion}

We developed a respiration-leading stuffed toy for children to encourage them to learn the respiration method that helps them relax. The respiration wave was measured by the sensing device, and the child's respiration could be led by the moving built-in device in the stuffed toy. Our results suggest that the change in mean HR of children who could synchronize their respiration under the respiration-leading condition was smaller compared with the hugging condition. This suggests that the relaxation effect is the direct result of the respiration-leading function of the stuffed toy, rather than simply hugging effect. Application of this toy in PTSD patients is expected in the future.

\section{Acknowledgement}

We thank the participants of this experiment, and EQW-
EL Co., Ltd. who cooperated with this experiment. This paper is a part of my doctoral thesis that can be accessed from the repository of Osaka Institute of Technology [19].

\section{Conflict of Interest}

The authors declare no conflict of interest.

\section{References}

1. Yamada F, Dodo N, Oono T, Hattori S: The effect of seismic intensity and sex difference on school-aged children's stress reactions after the Great Hansin-Awaji Earthquake. Jpn Soc Phys Anthropol. 4(1), pp. 23-28, 1997 (in Japanese).

2. Zucker T, Samuelson K, et al.: The effects of respiratory sinus arrhythmia biofeedback on heart rate variability and posttraumatic stress disorder symptoms - a pilot study. Appl Psychophysiol Biofeedback. 34(2), pp. 135-143, 2009.

3. Umezawa A: Self-regulation of respiratory activities in real life situations: Ambulatory monitoring of ventilation using respiratory movements. Jpn Soc Biofeedback Res. 24, pp. 22-27, 1997 (in Japanese).

4. Uratani H, Ohsuga M: A Study for the possibility of respiration leading by the respiration leading stuffed toy for children's relaxation. Jpn Soc Biofeedback Res. 41(1), pp. 19-26, 2014 (in Japanese).

5. Uratani H, Ohsuga M: Toward the development of a respiration leading stuffed toy for enhancement of children's relaxation. Jpn Soc Biofeedback Res. 39(2), pp. 77-84, 2012 (in Japanese).

6. Pastor M, Menendez F, et al.: The influence of respiration on biofeedback techniques. Appl Psychophysiol Biofeedback. 33, pp. 49-54, 2008.

7. Grant J, et al.: Inhalation-to-exhalation ratio affects HRV training success. Appl Psychophysiol Biofeedback. 35(1), pp. 181, 2010.

8. Lehrer PM, Vaschillo E, Vaschillo B: Resonant frequency biofeedback training to increase cardiac variability: rationale and manual for training. Appl Psychophysiol Biofeedback. 25(3), pp. 177-191, 2000.

9. Karavidas MK, Lehrer PM, Vaschillo E, et al.: Preliminary results of an open label study of heart rate variability biofeedback for the treatment of major depression. Appl Psychophysiol Biofeedback. 32, pp. 19-30, 2007

10. Siepmann M, Aykac V, et al.: A pilot study on the effects of heart rate variability biofeedback in patients with depression and in healthy subjects. Appl Psychophysiol Biofeedback. 33, pp. 195201, 2008.

11. Bernardi L, Porta C, et al.: Slow breathing increases arterial baroreflex sensitivity in patients with chronic heart failure. Circulation. 105, pp. 143-145, 2002.

12. Joseph CN, Porta C, et al.: Slow breathing improves arterial baroreflex sensitivity and decreases blood pressure in essential hypertension. Hypertension. 46, pp. 714-718, 2005.

13. Lin G, Xiang Q, et al.: Heart rate variability biofeedback decreases blood pressure in prehypertensive subjects by improving autonomic function and baroreflex. J Alternative Complementary Med. 18(2), pp. 143-152, 2012.

14. Lehrer PM: Biofeedback training to increase heart rate variability. In: Principles and Practices of Stress Management (3rd Ed) edited by Lehrer PM, Woolfolk RL and Sime WE. Guilford 
Press. pp. 227-248, 2007.

15. Uratani H, Yoshino K, Ohsuga M: Basic study on the most relaxing respiration period in children to aid the development of a respiration-leading stuffed toy. Proceedings of IEEE EMBC 2014, 2014.

16. Uratani H, Ohsuga M: Development of respiration leading stuffed toy using airbags for children. Jpn Ergonomics Soc. 51(6), pp. 428-434, 2015 (in Japanese).

17. Yamaguchi D, Ohsuga M: Development of a respiration leading application using music for relaxation. Proceedings of Human Interface Symposium 2014. pp. 297-300, 2014 (in Japanese).

18. Sumioka H, Nakae A, Kanai R, Ishiguro H: Huggable communication medium decreases cortisol levels. Sci Rep. 3, 3034, 2013.

19. Uratani H: Development and evaluation of a respiration-leading stuffed toy to learn relaxation method for children, 2016 (in Japanese). Doctoral thesis of Osaka Institute of Technology. http:// id.nii.ac.jp/1360/00000198/

\section{Hiroki Uratani}

Hiroki URATANI received his B.S. and M.S. degrees in Biological Science from Kyoto University in 1996 and 1999, respectively. He received his doctor's degree in engineering in Biomedical Engineering from Osaka Institute of Technology in 2016. He has been working as a chief researcher at

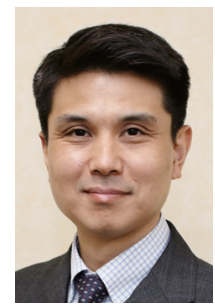
Neo-Education Science Research Institute of EQWEL Co., Ltd. since 2016. He is a member of Japanese Society for Medical and Biological Engineering, Physiological Psychology and Psychophysiology, and Japanese Society of Biofeedback Research.

\section{Mieko Ohsuga}

Mieko Ohsuga received her B.S. degree in Measurement and Instrumentation from Tokyo University in 1979 and received her doctor's degree in engineering from Tokyo University in 1994. She worked for Mitsubishi Electric Corporation 19792002 and moved to Osaka Institute of Technology.

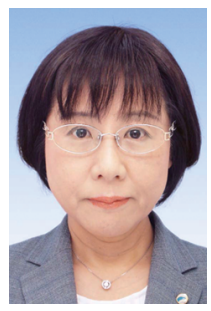
She is currently a professor of Department of Robotics, Faculty of Robotics \& Design. She is a member of IEEE EMBS, Japanese Society for Medical and Biological Engineering, Human Interface Society, Japan Ergonomics Society, etc. 